ANNUAL OF NAVIGATION 19/2012/part 2

10.2478/v10367-012-0026-4

SERGEY YAKUSHIN

State National Research Polytechnic University of Perm, Russia

\title{
SYNERGETIC CONCEPT \\ OF ALGORITHMS AUTONOMOUS INERTIAL NAVIGATION SYSTEMS
}

\begin{abstract}
Errors of INS output parameters lead to a positive feedback effect of errors and eventually to an even more dramatic increase in system errors. To reduce the impact of this problem on the error output parameters of INS, in this paper, we propose and study a new concept of constructing algorithms for autonomous INS, which is called as synergetic concept. In the paper the synergetic concept of inertial system's algorithm is presented and investigated by implementing its into strapdown inertial navigation system (SDINS).
\end{abstract}

\section{Keywords:}

Inertial Navigation System, Autonomous INS.

\section{INTRODUCTION}

It is well known, the schemes of the algorithms autonomous inertial navigation systems (INS) consists of two main sequential functional blocks, which complete the initial alignment and operating mode, including the calculation of the current position coordinates, velocity and the vehicle angular orientation parameters. To improve the accuracy of such systems, usually external information is used, and in this case, INS is integrated and non-autonomous.

The use of INS in autonomous mode is preferred for many applications of these systems, because in this case this system is the most simple and reliable. However, it is well known that errors of INS working in autonomous mode, have a general tendency to increase in low frequency. The reason for the growth of errors is in the influence of errors, respectively, in the mode of initial alignment, accelerometer and gyroscope errors in the dynamic operating conditions, as well as computational error processing algorithm for measurement information (mainly the integration errors of measurements from accelerometers). 
Errors of INS output parameters (calculated coordinates of current location, linear velocity of the object and its angular orientation parameters) lead to a positive feedback effect of errors and eventually to an even more dramatic increase in system errors. To reduce the impact of this problem on the error output parameters of INS, in this paper, we propose and study a new concept of constructing algorithms for autonomous INS, which is called as synergetic concept.

The purpose of the new concept of algorithm INS is to improve the accuracy of the system without some external measurement data and also without ZUPT-method correction. The essence of the synergetic concept is to fusion mode of current self-evaluation of the main parameters of INS and the conventional operating mode. In this scheme of the INS algorithm is achieved an accuracy improvement of the system on a moving vehicle by using only internal algorithmic (autonomous) capabilities of INS. The idea of developing this concept is based, in particular, on the results of the analysis of the application developed by the author of a new autonomous method of initial alignment of INS, which is implemented on the vehicle that performs a spatial and angular motion [1].

The new concept of the algorithm of inertial navigation system has an universal character and can be used both for the gimbaled and for the strapdown systems. At present, a class of strapdown inertial navigation systems (SDINS), and also their subclass - strapdown orientation systems has more and more wide application in many areas of use of modern mobile objects. Thus one of the primary goals of improvement of these systems is increase of their accuracy and reliability of operation. In this paper we consider the synergetic concept of algorithms INS by implementing of the strapdown inertial navigation system (SDINS).

\section{THE NEW METHOD}

The most difficult mode of SDINS is the mode of initial alignment, however the system errors depend largely on accuracy of its realization also in operational mode. The character and influence of errors of an initial alignment on target parameters SDINS are well-known, and they are the proof of importance of this mode for achievement of required precision of inertial system in operational mode. At present, aware of the various methods of initial alignment of SDINS on the stationary and moving base are known. In this paper, the mode of autonomous initial alignment of SDINS on the moving vehicle is discussed.

The main purpose of the initial alignment, realized in this concept is to determine the attitude angles of the vehicle relative to the local level frames (true azimuth 
(heading), pitch, roll, yaw). It coincides with the traditional task of initial alignment for SDINS on a moving base. In contrast to the known methods of solving this problem in presented method the use of any external information for the initial alignment is not assumed. The algorithmic process of initial alignment begins under the conditions for the unknown (arbitrary) initial orientation of measuring unit relative to the local level frames and direction to true north, and also in the absence the data about the linear velocity of the motion of vehicle relative to the Earth.

The algorithm of this method is based only on the transformations of measurable data from the SDINS gyroscopes and accelerometers. The method essence consists in an adequate transferring of ideology of an initial alignment of the platform inertial navigation system as applied to SDINS and taking into account features of its operation.

In the SDINS there is no gyroscopic stabilized platform which physically models usually local level frame, and accelerometers and gyroscopes are mounted directly on the base. These sensors, unlike platform system, are not isolated from angular motion of the base and that therefore complicates procedure of an initial alignment. In our method the gyroscopic stabilized platform is replaced with the virtual platform which angular position relative to local level frame is simulated by means of a computational algorithm.

The initial alignment process includes the concurrently running leveling and gyrocompassing of the virtual platform [1]. For an angular position estimation of the virtual platform relative to the horizontal plane (that is necessary for it leveling) we will use measuring signals from accelerometers, transformed to the virtual platform frame. The direction cosine matrix $A$ between the virtual platform frame and the body frame we will calculate by the numerical integration of Poisson matrix differential equation:

$$
\dot{A}=\omega_{G} A-A \omega_{P},
$$

where $\omega_{G}$ and $\omega_{V P}$ are the skew symmetric matrixes of the angular rates sensed from gyroscopes, and computed from the virtual platform respectively.

The leveling process of the virtual platform is realized by means of PID-regulators with use of as minimized angular parameters the calculated mismatch of the virtual platform frame relative to the true horizontal plane, i.e. with pitch $\vartheta_{V P}$ and roll $\gamma_{V P}$ angles of the virtual platform. Integral components of the virtual platform control $I_{\vartheta}, I_{\gamma}$ (in pitch and roll control channel respectively) in quasisteady state of 
the gyrocompassing mode can be used for heading calculation $\psi_{V P}$ of the virtual platform according to expression

$$
\psi_{V P}=-\tan ^{-1}\left(I_{\vartheta} / I_{\gamma}\right) .
$$

Heading of the virtual platform $\psi_{V P B}$ relative to longitudinal axis of the body frame we will obtain under the information on elements of calculated matrix $A$ from (1)

$$
\psi_{V P B}=\tan ^{-1}\left(a_{21} / a_{22}\right) .
$$

Then, heading of the base (vehicle) $\psi_{B}$ will be defined by expression

$$
\psi_{B}=\psi_{V P}+\psi_{V P B} .
$$

For the subsequent damping angular rates of the virtual platform in a control mode it is advisably to use low-pass filter. In this paper we consider a simple first-order low-pass filter in the form of aperiodic transfer function. At the time of completion gyrocompassing of SDINS according to the chosen for this criterion (for example, to achieve acceptable values of the standard deviation of the gyrocompassing course of virtual platform or course of the base), we define the heading of the base, according to (4), and compute the pitch and roll angles of the base by formulas

$$
\vartheta=\sin ^{-1}\left(a_{23}\right), \gamma=-\tan ^{-1}\left(a_{13} / a_{33}\right) .
$$

Then we can go into operational mode of the SDINS.

It should be noted that under certain conditions (such as a relative unknown to the allowable limit of the linear velocity of the object, the allowable limit of the angular movements of the object), an autonomous process of initial alignment is stable and provides estimates of the convergence of the angular orientation of a moving object. At the same time, a distinctive feature of the autonomous operation mode is its fundamental instability in the calculation of the output parameters of the inertial system.

Synergetic concept of algorithms INS is to share the opportunities of both modes in a single algorithmic scheme. This scheme allows the algorithm of INS in general, increasing the stability of the regime by extending the initial alignment also in operational mode while improving the accuracy due to the fact that the data will reduce uncertainty about the relative linear velocities of the object by changing the information on these velocities derived from the traditional algorithm of SDINS. At the initial stage of the synergetic algorithm can be with maximum robustness to believe 
the unknown relative linear velocity of the object to be zero. Of course, to determine these rates, such as the sea, we can use different types of autonomous lag and thereby improve the accuracy of the initial alignment on the first stage of the algorithm of INS. However, in this paper, we deliberately complicate the delivery of objectives in order to more clearly show the possibility of a new concept in the most difficult conditions, to evaluate the efficiency and quality of the proposed concept of a scheme for high-precision modeling of the marine SDINS based on the use of this synergetic concept in view of the characteristic errors of inertial sensor - gyroscopes and accelerometers.

\section{THE MODEL OF SIMULATION}

The modeling scheme includes the following main components:

— simulation model of the complex spatial angular and linear moving of the vehicle;

- calculation of measuring signals from the accelerometers and the gyroscopes (angular rate sensors) for the simulated motion of the vehicle, including their typical deterministic and random errors (bias, scale factor, random drift, etc);

- shaping the loops of processing measurement data on the basis of matrix transformation and elements of PID-regulators with an appropriate logic of their operation - for the initial alignment process;

- calculation of initial alignment errors due to various factors;

- conventional algorithm of SDINS;

- synergistic union of an initial alignment mode and the standard algorithm for operating mode of the SDINS;

- determination of output parameter errors of the conventional algorithm SDINS and synergetic algorithm.

The main object for which we will simulate the operation of the system, we consider a hypothetical naval object with a small relative velocity — up to $15 \mathrm{~m} / \mathrm{s}$. Let's present the description of the specified research blocks of SDINS initial alignment method. As mathematical model of complicated space movement of a marine vessel with variable horizontal velocity relative to the Earth $V$, and also with time variables heading $\psi$, pitch $\vartheta$, roll $\gamma$ and vertical velocity $V_{z}$ we will consider the following equations:

$$
\begin{aligned}
& V=V_{0}+A_{V} \sin \left(\omega_{V} t\right) ; \\
& \psi=\psi_{0}+A_{\psi} \sin \left(\omega_{\psi} t\right) ;
\end{aligned}
$$




$$
\begin{gathered}
V_{N}=V \cos \psi ; \\
V_{E}=V \sin \psi ; \\
V_{Z}=A_{V Z} \sin \left(\omega_{V Z} t\right) ; \\
\vartheta=\vartheta_{0}+A_{\vartheta} \sin \left(\omega_{\vartheta} t\right) ; \\
\gamma=\gamma_{0}+A_{\gamma} \sin \left(\omega_{\gamma} t\right) .
\end{gathered}
$$

Local latitude $\varphi$ and longitude $\lambda$ of vessel position, according to simulated moving in relation to the Earth will change in following way:

$$
\begin{gathered}
\dot{\varphi}=V_{N} / R_{2} ; \\
\dot{\lambda}=V_{E} /\left(R_{1} \cos (\varphi)\right),
\end{gathered}
$$

where $R_{1}$ and $R_{2}$ - the Earth radiuses of the reference ellipsoid, defined by well-known formulas from geodesy.

According to mathematical model of marine vessel moving (6-14) we will write down expressions for definition of measured accelerations $A_{E}, A_{N}, A_{Z}$ in local level frame ENZ which axes points to the east, north and to the local vertical upwards respectively

$$
\begin{gathered}
A_{E}=\dot{V}_{E}-2 V_{N} \Omega \sin \varphi-V_{E} V_{N} \tan \varphi / R_{1}+V_{Z}\left(\Omega \cos \varphi+V_{E} / R_{1}\right) ; \\
A_{N}=\dot{V}_{N}+2 V_{E} \Omega \sin \varphi+V_{E}^{2} \tan \varphi / R_{1}+V_{Z} V_{N} / R_{2} ; \\
A_{Z}=-V_{N}^{2} / R_{2}-V_{E}^{2} / R_{1}-2 V_{E} \Omega \cos \varphi+g+\dot{V}_{Z}
\end{gathered}
$$

The measured accelerations vector in the body frame $A_{b}$ we will obtain by the following transformation

$$
A_{b}=M A_{n},
$$

where $M$ - direction cosine matrix between the body frame and local level frame.

The elements of matrix $M$ we will calculate on preset values of orientation angles defined by the equations (7), (11), (12)

$$
\begin{aligned}
& m_{11}=C_{\gamma} C_{\psi}-S_{\gamma} S_{\vartheta} S_{\psi} ; \\
& m_{12}=C_{\gamma} S_{\psi}+S_{\gamma} S_{\vartheta} C_{\psi} ;
\end{aligned}
$$




$$
\begin{gathered}
m_{13}=-S_{\gamma} C_{\vartheta} ; \\
m_{21}=-C_{\vartheta} S_{\psi} ; \\
m_{22}=C_{\vartheta} C_{\psi} ; \\
m_{23}=S_{\vartheta} ; \\
m_{31}=S_{\gamma} C_{\psi}+C_{\gamma} S_{\vartheta} S_{\psi} ; \\
m_{32}=S_{\gamma} S_{\psi}-C_{\gamma} S_{\vartheta} C_{\psi} ; \\
m_{33}=C_{\gamma} C_{\vartheta},
\end{gathered}
$$

where designation $C_{()}=\cos (), S_{()}=\sin ()$ is used.

Now, if we add to the vector of accelerations $A_{b}$ an accelerometer errors, we will obtain a simulated accelerometer signals in the body frame which we will use further in simulation program by modelling of the SDINS initial alignment. In addition, it is necessary to execute imitation of measuring signals from gyroscopes for the moving preset values of the vessel in space.

Let's consider as measuring signals from gyroscopes (angular rate sensors) the corresponding angular rates in axes of the body frame. In this case, taking into account change of orientation of the body frame relative to the local level frame, described by well-known Euler transformation, we will obtain following expressions for these angular rates

$$
\begin{gathered}
\omega_{b x}=\dot{\vartheta} C_{\gamma}-\dot{\psi} C_{\vartheta} S_{\gamma}+\Omega_{\gamma}\left(S_{\psi} C_{\gamma}+C_{\psi} S_{\vartheta} S_{\gamma}\right)-\Omega_{Z} C_{\vartheta} S_{\gamma}+\Omega_{X}\left(C_{\psi} C_{\gamma}-S_{\psi} S_{\vartheta} S_{\gamma}\right) ;( \\
\omega_{b y}=\dot{\gamma}+\dot{\psi} S_{\vartheta}+\Omega_{\gamma} C_{\psi} C_{\vartheta}+\Omega_{Z} S_{\vartheta}-\Omega_{X} S_{\psi} C_{\vartheta} \\
\omega_{b z}=\dot{\psi} C_{\vartheta} C_{\gamma}+\dot{\vartheta} S_{\gamma}+\Omega_{\gamma}\left(S_{\psi} S_{\gamma}-C_{\psi} C_{\gamma} S_{\vartheta}\right)+\Omega_{Z} C_{\vartheta} C_{\gamma}+\Omega_{X}\left(S_{\psi} S_{\vartheta} C_{\gamma}+C_{\psi} S_{\gamma}\right) ;(30)
\end{gathered}
$$

where $\Omega_{X}=-\dot{\varphi}, \quad \Omega_{Y}=(\Omega+\dot{\lambda}) \cos \varphi, \quad \Omega_{Z}=(\Omega+\dot{\lambda}) \sin \varphi$.

Adding the corresponding errors to expressions from (28) to (30), we will obtain simulated signals of angular rate sensors in the body frame which further will be also as well as simulated signals of accelerometers, to use in simulation program by modelling of the SDINS initial alignment.

The operational algorithm of the PID-regulators used in a control loop of the virtual platform, looks like 


$$
\begin{gathered}
\omega_{V P x}=-k_{1 x} \vartheta_{V P}-k_{2 x} l_{\vartheta}-k_{3 x} \dot{\vartheta}_{V P} ; \\
\omega_{V P y}=-k_{1 y} \gamma_{V P}-k_{2 y} l_{\gamma}-k_{3 y} \dot{\gamma}_{V P} ; \\
\omega_{V P z}=\Omega \sin \varphi .
\end{gathered}
$$

On the basis of simulation results for the contributed method of an initial alignment it is obtained, that satisfactory results on alignment accuracy are provided, in particular, at following PID-regulators parameter values and logic of their switching.

Table 1. Parameters of PID-regulators [own study]

\begin{tabular}{|l|c|c|c|c|c|c|}
\hline & $k_{1 x}$ & $k_{2 x}$ & $k_{3 x}$ & $k_{1 y}$ & $k_{2 y}$ & $k_{3 y}$ \\
\hline $0 \leq t \leq 200 s$ & 0.9 & 0.01 & 0.9 & 0.9 & 0.01 & 0.9 \\
\hline$t>200 s$ & 0.01 & 0.00002 & 0.005 & 0.01 & 0.00002 & 0.005 \\
\hline
\end{tabular}

We show now the main results of sequential simulation mode of the initial alignment and operational (inertial) mode of SDINS, which will continue to be thought a foundation to support the concept of the synergetic algorithm of autonomous inertial systems. The sequence of modes of simulation is that the simulation is carried out first mode of SDINS initial alignment on a moving object, the linear velocity and angular parameters are unknown, but is limited to the upper range, and then the data on the angular orientation, the system calculated at the end of the initial alignment, transferred to the operating mode of the conventional algorithm of SDINS. Thus, we simulate normal system operation, carrying out the initial alignment on a moving base.

\section{PARAMETERS OF SIMULATED MOVEMENT OF VESSEL}

By simulation of initial alignment algorithm the various movement parameters of the marine vessel, and also various sources of errors of SDINS sensors, which can take place in practice, were considered. One of variants of these parameters is presented below:

— time sampling: $100 \mathrm{~Hz}$;

- horizontal velocity: $V_{0}=10 \mathrm{~m} / \mathrm{s}, A_{V}=0.2 \mathrm{~m} / \mathrm{s}, \omega_{V}=1.27 \mathrm{~s}^{-1}$;

- vertical velocity: $A_{V Z}=1 \mathrm{~m} / \mathrm{s}, \omega_{V Z}=1 \mathrm{~s}^{-1}$;

- heading parameters: $\psi_{0}=54^{\circ}, A_{\psi}=10^{\circ}, \omega_{\psi}=1 \mathrm{~s}^{-1}$;

— pitch parameters (trim angle): $\vartheta_{0}=5^{\circ}, A_{\vartheta}=10^{\circ}, \omega_{\vartheta}=1 \mathrm{~s}^{-1}$; 
— roll parameters: $\gamma_{0}=13^{\circ}, A_{\gamma}=8^{\circ}, \omega_{\gamma}=1 s^{-1}$;

— duration of initial alignment $-2500 s$;

- accelerometer biases $-0.001 \mathrm{~m} / \mathrm{s}^{2}$;

— accelerometer random drift $-0.1 \mathrm{mg}(3 \sigma)$;

- gyroscope biases $-0.05^{\circ} / \mathrm{h}$;

- gyroscope random drift $-0.05^{\circ} / h(3 \sigma)$.

For this version of the algorithm parameters of the study of initial alignment and operating mode of SDINS, following results were obtained by calculating the errors of azimuth, pitch, roll, and also the latitude and longitude of the simulated motion of the vessel (fig. 1-5), changing over time. The total simulation time (indicated on the drawings in seconds) is the sum of the initial alignment time (the first mode) and operating mode time of SDINS, equal to two hours.

In fig. 1-3 the error of orientation angle (in degrees) computed in the initial alignment mode and in a conventional algorithm for SDINS (for the first mode solid lines, for the second mode - in the form of points) is presented. In Fig. 4-5 the error of the coordinates, respectively, in latitude and in longitude (in meters, with a scale factor 10000) is presented.

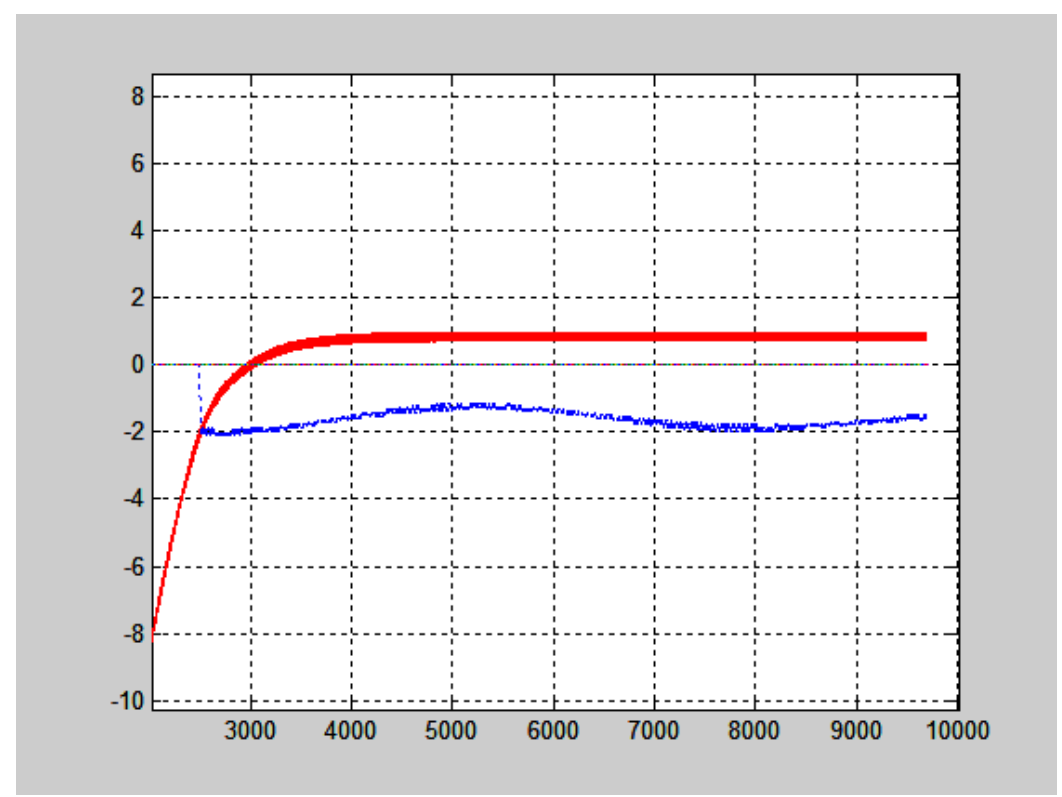

Fig. 1. Errors by azimuth calculation in the initial alignment mode, and after switch to operational mode of SDINS [own study] 


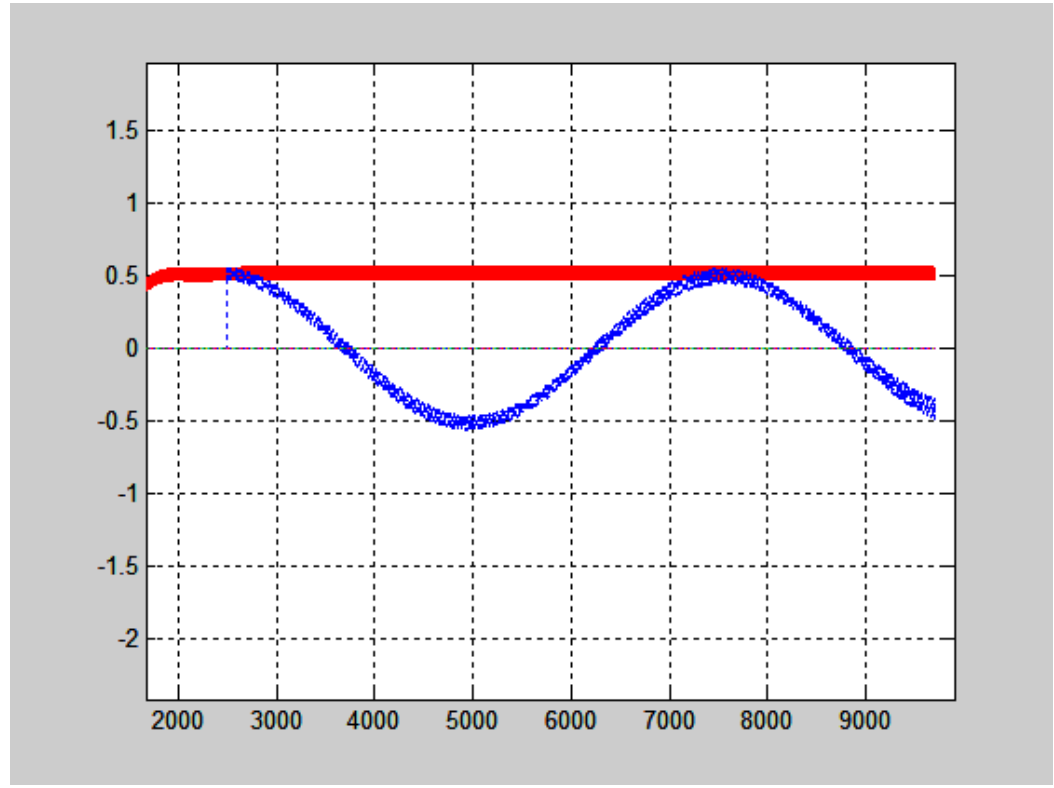

Fig. 2. Errors by roll angle calculation in the initial alignment mode, and after switch to operational mode of SDINS [own study]

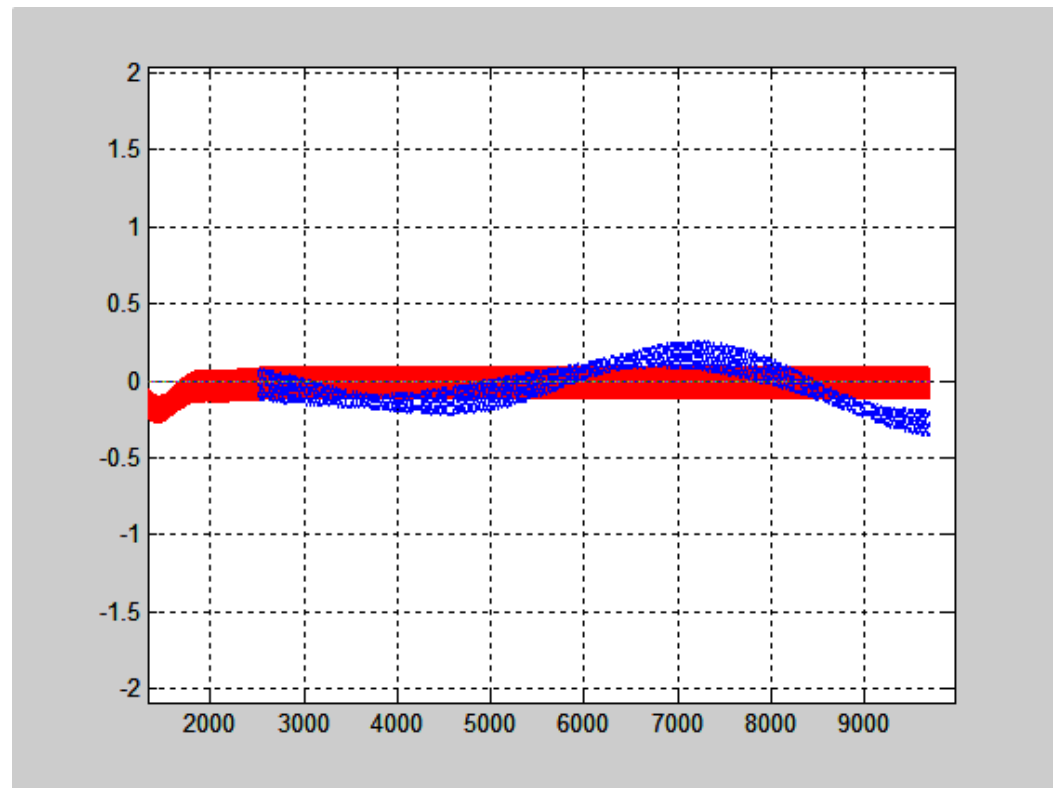

Fig. 3. Errors by pitch angle calculation in the initial alignment mode, and after switch to operational mode of SDINS [own study] 


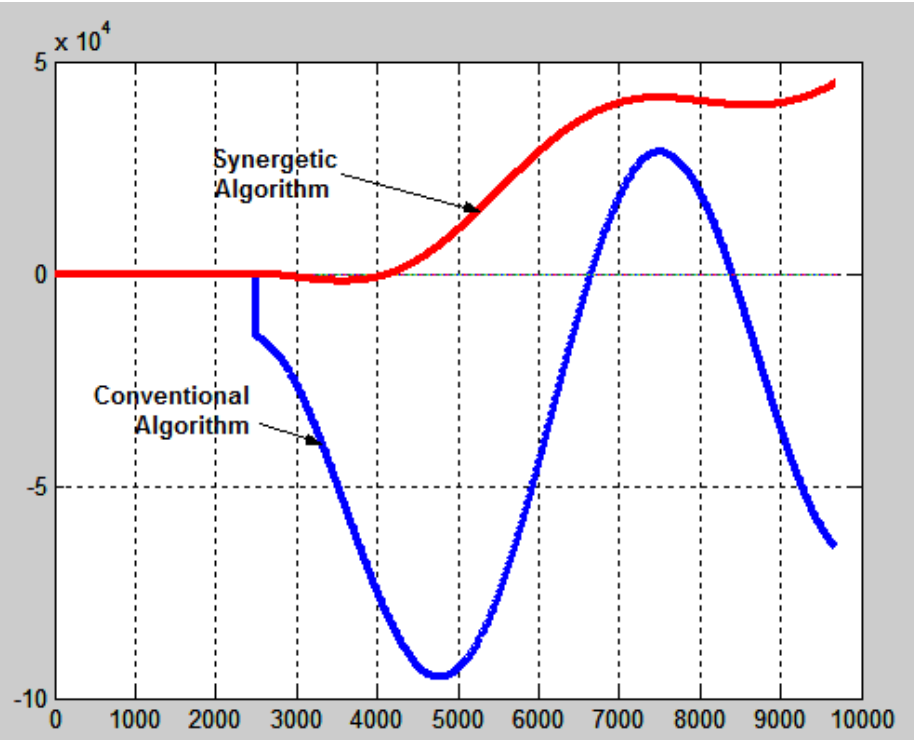

Fig. 4. Errors by latitude calculation after switch to the operational mode of SDINS [own study]

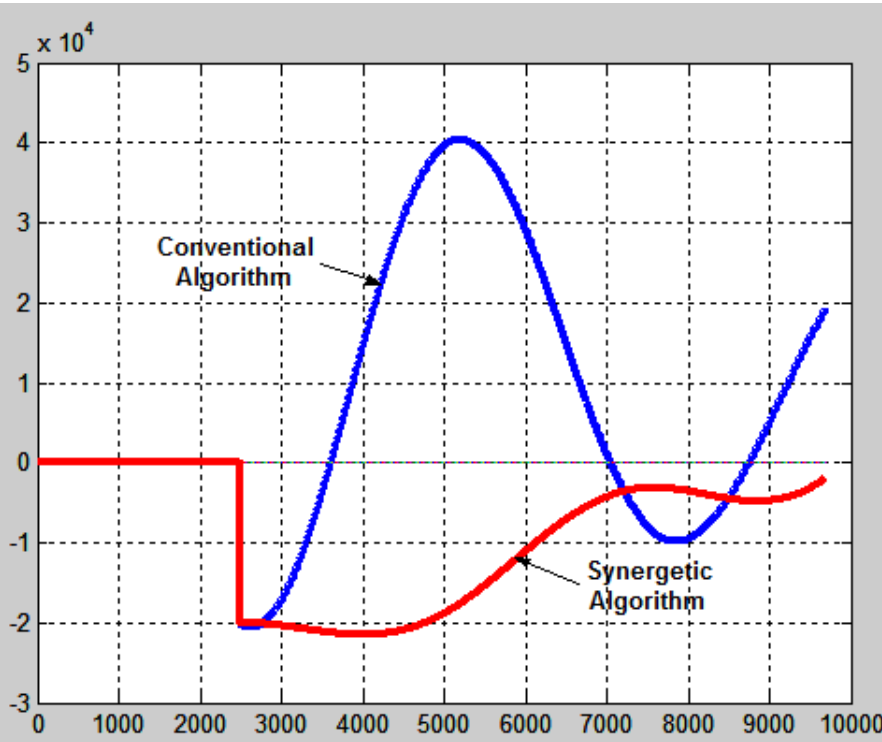

Fig. 5. Errors by longitude calculation after switch to the operational mode of SDINS [own study] 


\section{CONCLUSIONS}

The presented results are characteristic enough for parametres of movement of vessel and for the given class and level sensor's errors. This type of plots has a small changes caused by insignificant (in 1,5-2 times) variation of the vessel motion parametres and sensor errors concerning numerical data resulted above. Based on these results and other similar studies it is possible to make the following conclusions:

- character of errors of the first and second modes essentially differ; in the first case they look like constants, and in the second case as a variable processes in time; well-known, that errors of operating mode of SDINS have fluctuations with the Schuler's period that is shown also in our modelling;

- at sufficient time of an initial alignment the second mode as a whole on level of errors will be about one order with errors of the first mode during the limited operating time;

- at reduction of duration of the first mode, the errors of convetional algorithm SDINS will tend to faster increase, and parallel continuation of the first mode (after formal 'end' of an initial alignment) will be characterised, on the contrary, by reduction of errors of calculation of angular orientation of the vessel.

It is possible to explain last conclusion to that errors in angular orientation of a virtual platform at the moment of formal end of the first mode are transferred into standard algorithm of SDINS, leading to known accumulation of errors of calculation of the output parametres caused by errors of an initial alignment. Thus the basic source of increase in errors in the second mode is double integration of components of a vector of the gravity acceleration, having projections to the directions of computing axes of the local level frames in algorithm of SDINS.

If to take advantage of both modes in a single processing measurement data, it is possible to achieve a synergistic effect, the use of which will improve the accuracy of SDINS. The scheme proposed synergetic concept of constructing algorithms of INS is the following:

- operation of the first and second modes to make parallel (instead of serial);

- the parameters of angular motion of the virtual platform (first mode), calculated as accurately as possible, to use in conventional algorithm of SDINS as calculated angular velocity of the local level frames;

- based on the fact that the errors on the pitch and roll of virtual platforms tend to constant values, to specify their values by bringing information about the error 
calculating the height of the object according to the convetional marine SDINS algorithm.

Calculation of constant errors in angular position of the virtual platforms in relation to the horizon plane can be carried out under following formulas based on the definition of height as double integral of the vertical acceleration:

$$
\Delta \vartheta \approx-\frac{2 \cdot\left(\Delta h_{1}-\Delta h\right)}{g t^{2} \delta \vartheta}
$$

where $\Delta h_{1}-\Delta h-$ an increment of the height calculated in algorithm of SDINS for the time $t$, during which to the calculated value of the pitch angle the fixed value $\delta \vartheta$ (under condition $\delta \gamma=0$ ) is added.

Similarly,

$$
\Delta \gamma \approx-\frac{2 \cdot\left(\Delta h_{1}-\Delta h\right)}{g t^{2} \delta \gamma}
$$

As shown by simulation results, the implementation of the synergetic concept of algorithm INS is universal and can be used for different types of systems (both platform and strapdown) and improves the accuracy of these systems. The level of increase of accuracy in autonomes mode is determined by the magnitude and rate of change of linear and angular motion of the object, as well as errors in inertial sensors.

As prospects of development of this concept is supposed the solution of problems connected with optimisation of control of virtual platform in order to reduce the transition time of its angular stabilisation in navigational frames and with improvement of quality of its control.

\section{REFERENCES}

[1] Yakushin S. M., Novel Method for Autonomous Initial Alignment of Strapdown Inertial Navigation System on Moving Base, The European Navigation Conference on Global Navigation Satellite Systems, Braunschweig, Germany, 2010.

Received May 2012

Reviewed September 2012 